\title{
Buck converter design for Photovoltaic generators with supercapacitor energy storage
}

\author{
Dariga Meekhun ${ }^{1,2}$, Vincent Boitier ${ }^{1,2}$, Jean-Marie Dilhac ${ }^{1,2}$ \\ Stéphane Petibon ${ }^{1,2}$, Corinne Alonso ${ }^{1,2}$ and Bruno Estibals ${ }^{1,2}$ \\ ${ }^{1}$ CNRS; LAAS; 7, avenue du Colonel Roche F-31077 Toulouse, France \\ ${ }^{2}$ Université de Toulouse; UPS, INSA, INP, ISAE; LAAS F-31077 Toulouse, France \\ Email: dmeekhun@gmail.com, Telephone number: (335) 61336837
}

\begin{abstract}
In Maximum Power Point (MPP) applications for photovoltaic generators, the DC-DC tracker converter is as important as the MPP tracking algorithm. The purpose of this work is to present the design methodology of a buck converter applied in maximum power point tracking. In this paper we also compare Hill-climbing and Fractional Voc MPPT efficiency. The MPPT algorithms were implemented with an autonomous DC/DC converter. It allows to determine their real advantages and disadvantages. Finally, our system will be used to charge supercapacitors. The most significant advantage supercapacitors have over batteries is their ability to be charged and discharged continuously without degradation and their low operating temperature $\left(-40^{\circ} \mathrm{C}\right)$.
\end{abstract}

\section{Key words}

BUCK converter, maximum power point tracking, MPPT, photovoltaic, $\mathrm{PV}$, supercapacitor

\section{Introduction}

The environmental problem is an enormous issue of today. Utilization of renewable energy is a good potential way to solve this problem. Therefore, clean-energy resources such as solar arrays, wind generators and others have attracted wide attention in both research and industry fields. However, solar energy attracts much attention because it can be used for powering many types of appliances. A photovoltaic (PV) energy conversion system may employ a DC to DC convertor including a Maximum Power Point Tracking (MPPT) function. It allows the PV array to transfer the maximum available energy to the load. As mentioned above, this paper proposes a design methodology of an autonomous buck converter with the maximum input power at 7 watts. We finally present in this paper the DC/DC converter efficiency implemented with MPPT algorithms (Hill-climbing and Fractional Voc). The approach presented in this paper is designed for charging supercapacitors.

\section{Related work and Background}

Several DC/DC converters for photovoltaic generators have been proposed in literatures. In [1], the energy efficiency between buck and boost converters is compared. It is shown that an MPPT based on the Boost converter configuration results in higher efficiency, thus better utilizing of the solar cells. In [2], the DC-DC Buck, Boost, Buck-Boost, Cúk, Sepic and Zeta converters are analyzed in order to determine which one is more proper to be applied as Maximum Power Point Tracker (MPPT). However, the simulation results demonstrated that, under low radiation, Boost converter has a poor tracking behavior. A development of a microcontroller-based boost converter for photovoltaic system is presented in [3]. The converter is designed to step up solar panel voltage to a stable output without storage elements such as battery. A Buck converter used to enhance the energy conversion efficiency in PV systems is analyzed and practically evaluated in [4-6]. The Buck converters presented in literatures are used to charge batteries. Nevertheless, by removing the battery altogether and storing energy solely in the supercapacitors, there is now a viable option for achieving long-life operation of any systems. In addition, charging a supercapacitor and drawing maximum solar power are both areas of active research. However, based on the authors' knowledge, only a publication is presented in [7]. The novelty of this system lies in the pulsed frequency modulated converter and open-circuit solar voltage method for maximum power point tracking, enabling the solar cell to efficiently charge the supercapacitors and power the load. However the system is very complicated to implement. Accordingly, we present in this paper a simple Buck converter that cans efficiency charge supercapacitors. However, in order to have high efficiency DC/DC converter, developers have to select appropriate components. Therefore we present in this paper how to select appropriate component with buck converter. Different MPPT algorithms are finally also compared. 


\section{BUCK converter Principle}

The DC-DC Buck converter was designed to process power from 1 to 7 watts. Fig. 1 is a simplified schematic diagram of the prototype DC/DC buck converter.

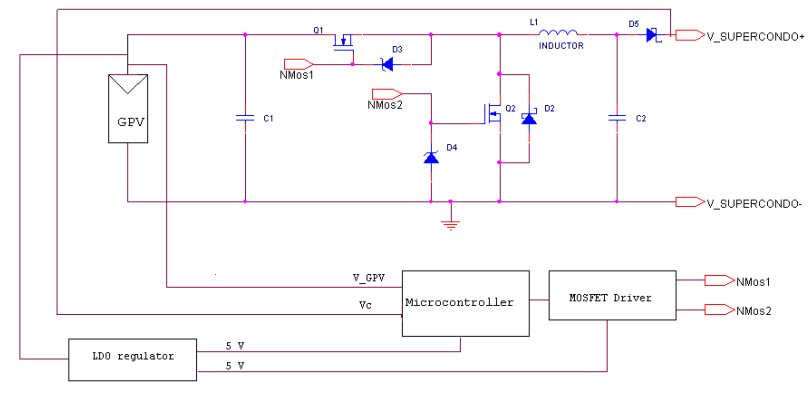

Fig. 1. Buck converter proposed to charge supercapacitors

Our proposed converter is a synchronous Buck converter. It is a modified version of the basic buck converter circuit topology in which the Schottky diode, D2, is connected in parallel with a second MOSFET switch, Q2. This modification improves efficiency because the MOSFET conduction losses are very low compared to the Schottky diode voltage drop. In this paper, the nominal voltage of a solar panel $\left(\mathrm{V}_{\mathrm{IN}}\right)$ is usually 10 volts. It is converted to an output of 5 volts ( $\mathrm{V}_{\text {OUT }}$ ) to charge the supercapacitors. The switching frequency $\left(\mathrm{F}_{\mathrm{SW}}\right)$ is selected at $400 \mathrm{kHz}$ and the load current, $\left(\mathrm{I}_{\mathrm{L}}\right)$ is 0.9 A. The current ripple $\left(\Delta \mathrm{I}_{\mathrm{OUT}}\right)$ will be limited to $20 \%$ of maximum load. We defined the methodology to choose the components in fig.1 by following keys below.

Inductor, L1: The required inductor is determined by [8]

$$
L=\left(V_{I N}-V_{O U T}\right) \cdot\left(\frac{V_{O U T}}{V_{I N} \cdot F_{S W}}\right) / \Delta I_{O U T}
$$

In this work, the calculated inductor value is $35 \mu \mathrm{h}$. Nevertheless its power losses are related with copper losses and current flow through the inductor. We must consider a low core loss inductor to obtain good performance. A SRU1028-330Y of Bourns Inductor is chosen for our application.

High MOSFET Switch, Q1: The losses associated with MOSFETs can be divided into conduction and switching losses. For high MOSFET switch, switching losses mostly dominate its power dissipation. Switching losses occur on each on/off transition. Total gate charge, $\mathrm{Qg}$ is the charge required to turn the MOSFETs on and off under the specified operating conditions. Qg should then be small because, the lower the charge, the lower the gate drive current needed to achieve a given switching time [4, 9 , 10]. In our application, we choose a FDN357N NChannel transistor.

Low MOSFET Switch, Q2: Since the bottom MOSFET switches current from/to a paralleled diode, the voltage across the MOSFET is no more than $1 \mathrm{~V}$ during switching transition. As a result, its switching losses are negligible. Then losses of Low MOSFET switch depend mostly on the conduction losses. Conduction losses are related to the static drain source on resistance $\left(R_{D S(O N)}\right)$ of MOSFETs, and increase with the load current. The $\mathrm{R}_{\mathrm{DS}(\mathrm{ON})}$ should then be small compared at the same gate voltage $[4,9,10]$. In this paper, an IRF7459 N-Channel transistor is chosen.

Schottky Diode, D2: The schottky diode is used to conduct the current during the non-overlap time when both MOSFETs are turned off. To choose a schottky diode, the diode current $\left(I_{D}\right)$ has to be determined. We can calculate by the equation below [8]:

$I_{D}=\left(1-\frac{V_{O U T}}{V_{I N}}\right) \cdot I_{L}$

In addition, to improve performance, a diode having low reverse recovery time (trr) should be chosen. In this work, the diode's average current is equal $0.45 \mathrm{~A}$. Thus, a schottky diode, 10BQ030PBF, with the maximum average forward current of $1 \mathrm{~A}$ meets requirement. Moreover, its low forward voltage allows the converter improve efficiency and reduce noise.

Zener voltage regulator diode, D3 and D4: The MOSFETs have a Zener diode disposed between the gate and source. It is used for prevent the breakdown of the MOSFET caused by overvoltage and static electricity. For that, the reverse voltage of the chosen zener diode should be smaller than the MOSFET maximum gatesource voltage. In this paper, 1SMB5932BT3G 1SMB5927BT3G zener diodes are used as D3 and D4, respectively.

Input capacitor, $\mathbf{C 1}$ : The primary purpose of the input capacitor is to provide quickly energy to the power stage (Q1, Q2, L1,C2) when Q1 is closed and charges L1 during steady state operation [11]. The eq.3 will approximate the minimum capacitance required.

$C_{I N}=\frac{V_{\text {OUT }}}{V_{I N} \cdot F_{S W}} /\left(\left(\Delta V_{I N} / \Delta I_{I N}\right)-E S R\right)$

Eq. 3

In our application, we define acceptable input ripple voltage $\left(\Delta V_{I N}\right)$ at 0.2 volts. The worst case ripple current $\left(\Delta I_{I N}\right)$ on the input of a buck converter is about one half of the load current. Thus it is at $0.45 \mathrm{~A}$. The ESR is the Equivalent Series Resistance of capacitors. Since, ceramic capacitors have very low ESR, a KEMET ceramic capacitors, C1210C475K3RACTU is selected in our application. In addition low ESR can limit the amount of input ripple voltage.

Output capacitor, C2: We can calculate the required capacitance of the output capacitor with the equation below [8]. 


$$
C_{\text {OUT }}=\left(\Delta I_{\text {OUT }} \cdot \frac{V_{\text {OUT }}}{V_{I N} \cdot F_{S W}}\right) /\left(\Delta V_{\text {OUT }}-\left(\Delta I_{\text {OUT }} \cdot E S R\right)\right) \quad \text { Eq. } 4
$$

The desired output voltage ripple $\left(\Delta V_{\text {OUT }}\right)$ is defined as 50 $\mathrm{mV}$. Thus the output capacitor in our application has to be higher than $5 \mu \mathrm{F}$. We chose two KEMET ceramic capacitors, C1210C335K5RAC-TU, $3.3 \mu \mathrm{F}$ connected in parallele.

Schottky Diode, D5: The schottky diode is implemented in order to avoid reverse current from the supercapacitors. A schottky diode, 10BQ030PBF is chosen because of its low forward voltage.

We have already presented the methodology to choose component. However to increase performance and efficiency of power MOSFETs, a MOSFET driver Si9913 is used. The power level is just slightly increased by the power consumption of the driver IC, which is relatively small compared to the power loss associated by the total gate charge of the MOSFET. Another advantage is to ensure that both high-side and low-side MOSFETs are not turned on at the same time.

To allow solar panels operate at their maximum power point, the voltage and current are continuously monitored by a microcontroller and the duty cycle of the buck converter continuously adjusted to extract maximum power. However the MOSFETs driver and the microcontroller require a power supply of 5 volts. In order to make the system autonomous, a LDO voltage regulator, TPS71550, is connected between solar panels and the components shown in fig.1. Then solar cell's power output powers directly devices through the LDO voltage regulator.

The autonomous buck converter is presented in fig. 2 However, to charge the supercapacitors, the study of MPPT functions and supercapacitors voltage regulation is also important.

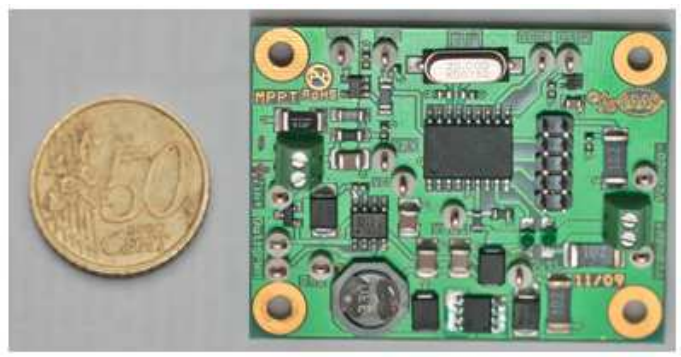

Fig.2 Autonomous buck converter

\section{Algorithm Implementation}

The different techniques of MPPT for photovoltaic arrays are presented in [12]. Among presented paper, our paper focuses on Hill-climbing and Fractional-Voc. Hillclimbing is interesting because of its high MPPT efficiency tracking. However two sensors (voltage and current) are required for this algorithm. Contrary to hillclimbing, fractional-Voc technique requires only a voltage sensor. In addition, it is easier to implement. However, it gives lower MPPT efficiency tracking the maximum power point than hill-climbing. To know their real advantages and disadvantages, both algorithms are implemented for performance evaluation.

We used a solar panel to charge ten Maxwell supercapacitors, PC10 (two PC10 in series mounted in parallel with another two PC10 in series). Each algorithm is implemented on the PIC18F1220 microcontroller. Fig. 3 shows the behavior of supercapacitors' charge characteristic with fractional-Voc algorithm. The open circuit voltage of solar panel is measured every 10 seconds.

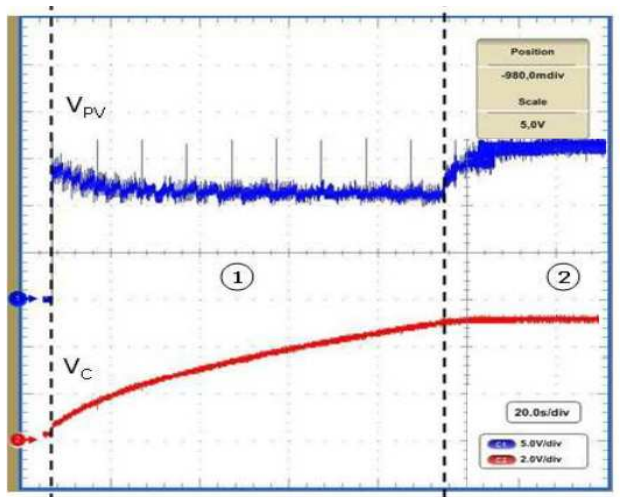

Fig. 3. Supercapacitor charge characteristic by fractional-Voc algorithm

Fig.3 shows that the autonomous buck converter with simple fractional-Voc algorithm can efficiently charge PC10 supercapacitors. When the supercapacitors' terminal voltage reaches 4.8 volts, the algorithm will move from MPPT mode to supercapacitors' voltage regulation mode.

The total efficiency $\left(\eta_{\text {TOTAL }}\right)$ that is the converter efficiency $\left(\eta_{\text {CONV }}\right)$ multiplied by the MPP tracking efficiency $\left(\eta_{\text {MPPT }}\right)$ is compared for different algorithms. The experiment is performed under different solar cell powers.

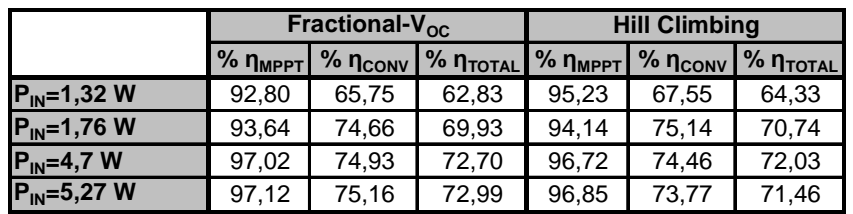

Tab.1 Efficiency Comparison between Fractional Open-Circuit Voltage technique and Hill-climbing technique

Tab.1 presents that the efficiency between Fractional Voc technique and Hill-climbing technique is approximately the same. We choose then Fractional Voc algorithm for our system because of fewer component requirement. It shows that the converter efficiency isn't significant different for different algorithms. Finally we compare, in table 2, the converter efficiency between our simply buck converter and the pulse width modulation converter presented in [7] at the same output voltage range. 


\begin{tabular}{|l|c|c|}
\hline \multicolumn{1}{|c|}{ DC/DC converter } & Vc (V) & Converter Efficiency (\%) \\
\hline PFM converter [7] & 1.7 to 2.5 & 40 to 50 \\
\hline Our Autonomous converter & 1.7 to 2.5 & 60 to 69 \\
\hline Our Autonomous converter & 2.5 to 5 & 69 to 78 \\
\hline
\end{tabular}

Tab.2 Converter Efficiency Comparison to charge supercapacitors

Tab. 2 shows that our simple converter has higher efficiency to charge supercapacitors.

However, the supercapacitor's voltage varies with the energy stored. While powering the load, the voltage of the supercapacitor is linear and drops from full voltage to zero volts. Nevertheless the load requires constant voltage. To fix output voltage, a regulator is inserted between the supercapacitors and the load.

\section{Conclusion and Discussion}

In conclusion, we presented in this paper the autonomous buck converter to charge the supercapacitors by the maximum available energy of PV array. To make the converter efficient, we simply have to choose appropriate components as presented above. In addition, two different algorithms are compared. Our system can prolong the lifetime of any system thanks to a battery-less system.

\section{References}

Glasner, I. and J. Appelbaum. Adventage of Boost vs. Buck Topology for Maximum power point tracker in Photovoltaic systems in Nineteenth Convention of Electrical and Electronics Engineers in Israel. 1996. Israel: IEEE.

[2] Coelho, R.F., F. Concer, and D.C. Martins. A study of the basic DC-DC converters applied in maximum power point tracking. in Brazilian Power Electronics Conference; COBEP '09. 2009

[3] Masri, S. and P.-W. Chan, Development of a Microcontroller-Based Boost Converter for Photovoltaic System. European Journal of Scientific Research 2010. 41(1): p. 39-47.

PETIBON, S., Nouvelles architectures distribuées de gestion et de conversion de l'énergie pour les applications photovoltaïques, in Génie Electrique. 2009, Université Toulouse III Paul sabatier: Toulouse. p. 143.

[5] Snyman, D.B. and J.H.R. Enslin. Novel technique for improved power conversion efficiency in $P V$ systems with battery back-up. in 13th International Telecommunications Energy Conference, 1991. INTELEC '91. 1991: IEEE.

[6] Snyman, D.B. and J.H.R. Enslin. Analysis and experimental evaluation of a new MPPT converter topology for $P V$ installations. in International Conference on Industrial Electronics, Control, Instrumentation, and Automation, 1992. Power Electronics and Motion Control. 1992: IEEE.
Simjie, F. and P. H.Chou. Everlast: Long-life, Supercapacitor-operated Wireless Sensor Node. in ISLPED. 2006. Tegernsee, Germany: IEEE.

] MicrochipTechnologyIncorporated, Buck Converter Design Example, B. Hutchings, Editor. 2006.

[9] Use Gate Charge to Design the Gate Drive Circuit for Power MOSFETs and IGBTs, in Application Note AN-944, InternationalRectifier, Editor.

[10] Sipex, SP6123, Low Voltage, Synchronous StepDown PWM Controller.

[11] AVNETelectronics. Input Capacitor Considerations for Buck DC Switching Power Supplies. Avnet Electronics Marketing Power eNewsletter [cited; Available from: http://www.em.avnet.com/ctf_shared/sta/df2df2 usa/PWRNL0907-article.pdf.

[12] Esram, T. and P.L. Chapman, Comparison of Photovoltaic Array Maximum Power Point Tracking Techniques. IEEE Transactions on Energy Conversion, 2007. 22(2): p. 439-449. 